\title{
Changes in Wall-Thickness Distribution by Hollow-Sinking
}

\author{
By Hiroshi Tanaka*
}

\begin{abstract}
Investigations are made on how the initial scatter in wall thickness existing along the transverse section of a tube will change when the tube is hollow-sunk through a die. The samples used are tubes of phosphor deoxidized copper and those of aluminium alloys. From the experimental results it turns out that the initial standard deviation in wall thickness, when not below $0.02 \mathrm{~mm}$, invariably decreases in its value. It is expected that tubes of a certain eccentricity could be improved if consecutive drawings are properly done.
\end{abstract}

(Received March 3, 1976)

\section{Introduction}

Investigations on the wall-thickness distributions along the transverse sections of industrially manufactured tubings always reveal more or less what is called "eccentricity", and when it is excessive, rejections or claims would be inevitable on the part of customers. Raw materials for manufacturing nonferrous tubings (copper, aluminium, etc.) are usually extruded tube shells (tube bloom), which are consecutively cold-worked to provide the required finished sizes.

The tube shells, as a rule, range from 70 to $90 \mathrm{~mm}$ in outer diameter, 7 to $9 \mathrm{~mm}$ (about $10 \%$ of the outer diameter) in wall thickness and 150 to $350 \mathrm{~kg}$ in copper weight. These are subsequently reduced both in diameter and wall thickness down to $(30 \sim 40 \phi) \times(1.5 \sim 2.0)$ $\mathrm{mm}$ in a single pass through the tube reducer, a rocking-type cold pilger roll, followed by consecutive drawings using winding type drawing machines, which are called "bull block".

However, the as-extruded tube shell is not quite uniform in wall thickness and sometimes of large eccentricity ${ }^{\dagger}$. This can be reduced to

* Department of Precision Mechanics, Faculty of Engineering, Tokai University, Tokyo 151, Japan.

$\dagger$ In practice, it is measured approximately by determining the great difference in wall thickness existing between diametrically opposite points in the same section. Sometimes it is defined as the percentage of the great difference to the specified wall thickness. as low as $5 \%$ through the tube reducer; it seems probably not easy to attain the more improved concentricity because of the inherent characteristics of the tool itself.

On the other hand, for the purpose of dispensing with the costly reducer, experiments have been conducted in the endevor to successfully extrude the tube shells, whose sizes are the same as those from the reducer and of the same order in wall-thickness deviation. Concerning this, efforts are also being made by the machine makers, but, to the author's knowledge, complete solutions have not been obtained as yet.

The persisting eccentricity is in general gradually corrected through successive drawing operations and when the finished size of the tubing is finally reached, its eccentricity may largely be eliminated; the more elimination is expected as the numbers of drawing pass are increased.

A great quantity of finished tubings are then delivered to the concomitant workers, where they are further processed, for instance, by bending, cutting, fin-tubing, etc., and where the accuracy in wall thickness is often of prime importance. Any relevant industrial standards specified for the eccentricity seem to be unable to cover all the demands of the customers.

The purpose of the present work is to bring out the dependence of hollow-sinking conditions on the changes arising in wall-thickness deviations (eccentricity). It is to be noted that even by the drawing with die and plug, a considerable proportion of reduction by sinking 
proceeds before the inner wall of tube touches the plug. Moreover, in the case of "bull block" with floating plug, many manufacturing plants utilize two dies in tandem, where the first die only reduces the outer diameter of the ingoing tubing (sinking) and the second one reduces both outer diameter and wall thickness (plug drawing).

Thus the hollow-sinking may be considered to play an important role in the wall-thickness distribution of the drawn tube. The influences of plug-drawing conditions on it will be reported shortly.

\section{Theoretical Background to the Thickness Change}

First, it seems necessary to refer to the wallthickness change by sinking as the eccentricity change is apparently attributable to this phenomenon.

When the tube is pulled through a converging die bore, the resulting hoop stress in the tube wall naturally works to increase its thickness even by a slight amount. The tube, when travelling towards the die exit, gradually suffers increasing axial tension until it is increased up to what is called pulling force at the die exit, thus some reduction in wall thickness being caused. The final wall thickness will increase or decrease according to which is more predominant over the other.

Chung $^{(1)}$ argued that at the die exit the tube suffered redundant axial tension owing to the unbending of the wall (directional change of the wall), which, in turn, gave rise to some additional thinning in thickness.

Experimental work by Okamoto ${ }^{(2)}$ showed that the maximum thickness occurred midway between incipient and final deformation (not strictly between die-entrance and die-exit), which might be well accounted for on the basis mentioned above.

Swift and Moore ${ }^{(3)(4)}$ predicted the change in wall thickness from the classical school of thought ("slab solution"); Flinn"(5) argued on the basis of dislocations and Avitzur ${ }^{(6)}$ by means of the upper bound solution assuming two velocity discontinuities on both sides of the die opening. Although they have con- tributed a good deal towards the explanation of the change in thickness, they are far from elucidating the dependence of other factors such as temper, kinds of material, etc. on it.

Figure 1 shows the change in wall thickness during the hollow-sinking of extruded aluminium alloy tubes (6063F) from $20 \phi \times 2 \mathrm{~mm}$ to $15 \mathrm{~mm} \phi$ o.d. (outer diameter) through conical dies of $6,13,20$ and $30^{\circ}$ in half angle, respectively.

In the figure "Entry" means a point where the surface of each tube first comes in touch with the die and "Exit" a point where the reduction in the conical bore terminates, i.e., the one at the entrance to the bearing bore (see Fig. 2).

It was observed, in any drawing conditions, that the tube suffered a slight increase in wall thickness prior to touching the drawing die and that the tube kept diminishing its wall thickness even after passing the die exit by a few $\mathrm{mm}$ beyond; the maximum thickness arose midway between the die entry and the die exit.

The above results obtained by the author were not quite different from those by other investigators. It is to be noted that the peak in the wall thickness occurred within the deforming zone and that when $\alpha$ was large enough, the final wall thickness became even smaller than that of the initial one.

Although not shown in the figure, the tube suffered reduction in o.d. during the whole stage of change in wall thickness; the reduction in o.d. began to occur slightly before the die entry and continued over some short distance beyond the die exit. The drawn tube exibited, therefore, a smaller o.d. than that

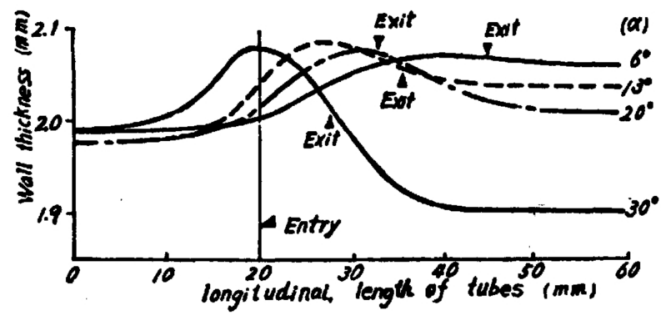

Fig. 1 Wall-thickness changes along longitudinal sections of the tubes including incipient and final deformations. 
of the die bore and the more so with increasing die taper.

\section{Experimental Procedure}

For the experiments concerned, use was made of commercially available tubes, and whose dimmensions were as follows:

Aluminium alloy tubes (6951) (Mg 0.6, Si 0.5, Cu $0.25 \%$ )

$17.0 \mathrm{~mm} \phi \times 1.3 \mathrm{~mm}$ Annealed and Hard

$19.0 \mathrm{~mm} \phi \times 1.3 \mathrm{~mm}$ Annealed

Aluminium alloy tube $(6063)(\mathrm{Mg} 0.5, \mathrm{Si}$

$0.4 \%$ )

$20.0 \mathrm{~mm} \phi \times 2.0 \mathrm{~mm} \quad$ As-extruded

Copper tubes (phosphor deoxidized)

$17.0 \mathrm{~mm} \phi \times 0.65 \mathrm{~mm}$ Hard and Annealed

$20.0 \mathrm{~mm} \phi \times 0.75 \mathrm{~mm}$ Hard and Annealed

$20.0 \mathrm{~mm} \phi \times 1.0 \mathrm{~mm}$ Hard

For experiments for sinking, each tube was cut to have a length of 1 meter and was drawn using a short experimental draw bench operated by an oil power unit, whose speed could be controlled between 0 and 6 meters per min. Use was made of the combinations of three kinds of die bore, 17.4, 15.0 and $13.0 \mathrm{~mm}$ in dia., with four kinds of half angle (semicone angle $\alpha$ ), 6, 13, 10 and $30^{\circ}$ for die taper (Fig. 2).

The dies were made of SKD-1 (C 2, Cr $13 \%$ ), the hardness lying between 56 and 59 HRc.

For all experiments only dry soap was used as a lubricant, the drawing speed ranging between 1 and 3 meters per min.

At points 1 to 8 and $1^{\prime}$ to $8^{\prime}$ of every halfdrawn sample (Fig. 3), the values in wall thickness and hardness (in micro Vickers) were measured within $100 \mathrm{~mm}$, so that the

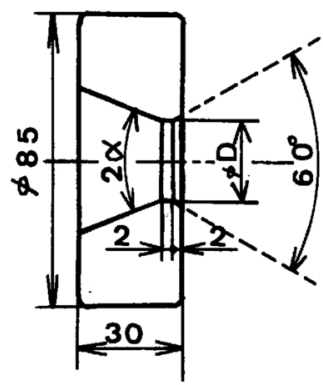

Fig. 2 Die-geometry used by the experiments. $D: \phi 17.4,15.0,13.0 \quad \alpha: 6,13,20,30^{\circ}$ Hardness: HRc 56 59.

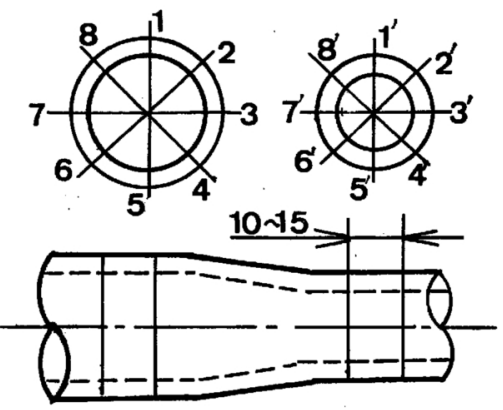

Fig. 3 Half drawn sample. Points 1-8 and 1'-8' indicate where wall thickness and hardness were measured.

values obtained before and after drawings can be correlated.

\section{Experimental Results and Discussion}

\section{Scatter in wall thickness before and after sinking}

Figures 4 and 5 show the wall-thickness distributions of phosphor deoxidized copper tubes before and after they were drawn, and Fig. 6 shows the results for aluminium alloy tubes of 69510 . In the figures, the mean values of wall thickness (mwt in the figures) together with the standard deviation $\sigma$ (both from 8 readings) are noted.

The increasing rate ( $\%$ change in the figures) denotes the difference of wall thickness at any point before and after drawing to the thickness before drawing in percent.

An interesting feature of the results is that the increasing rate in wall thickness after sinking is higher in the thinner part of the wall. It is seen that each run of the rate curve assumes nearly antipodal relation with that of the pre-drawn wall-thickness distribution curve, namely, if the unit length of the ordinate is adequately selected, two curves will run nearly symmetrically (mirror image).

The finding appears not very surprising since the strain in the thinner part of the wall should have been larger under constant hoop stress acting in the wall. As the thinner part of the wall is more increased in wall thickness than the thicker part, the sinking seems to have a function of flattening the wall-thickness dis- 

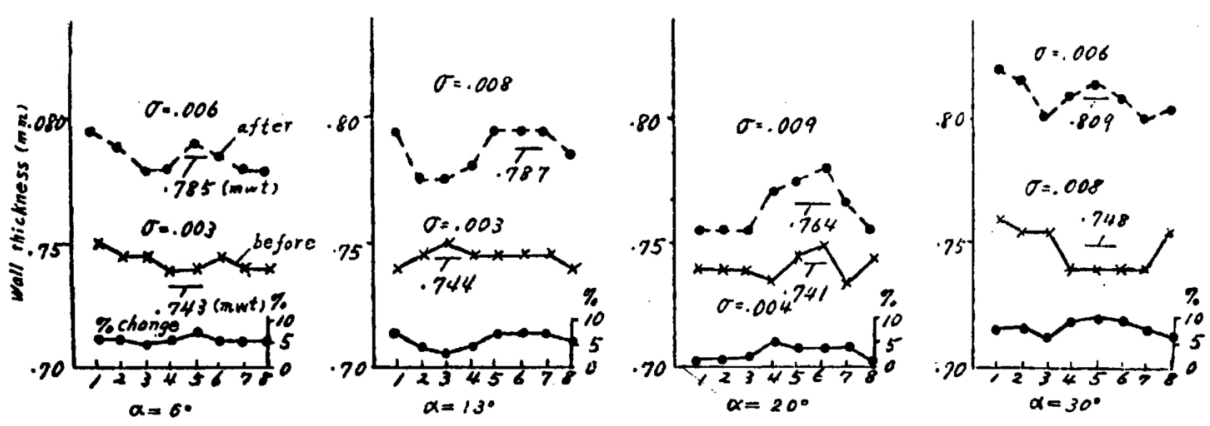

(a) $20.0 \phi \times 0.75 \longrightarrow 15.0 \mathrm{~mm} \phi 0 . d$.
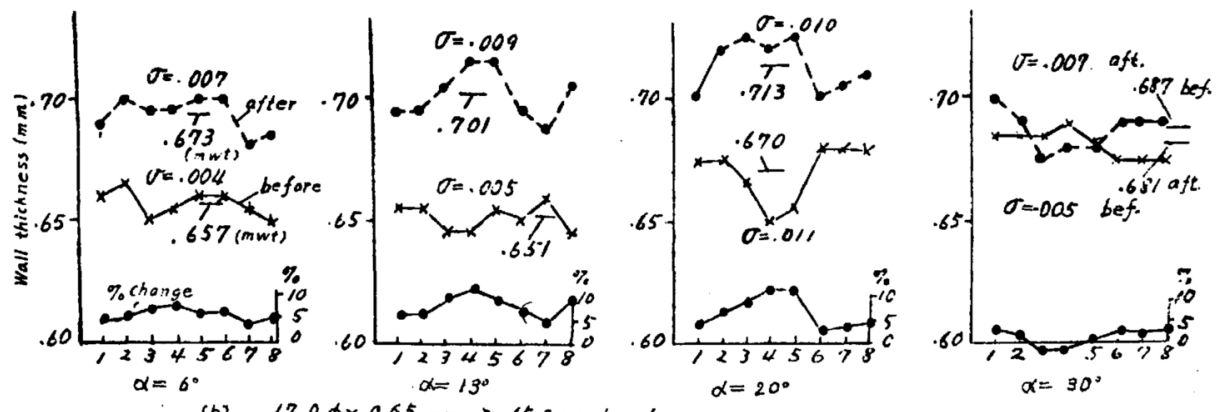

(b) $17.0 \phi \times 0.65 \longrightarrow 15.0 \mathrm{~mm} \phi 0 . \mathrm{d}$.
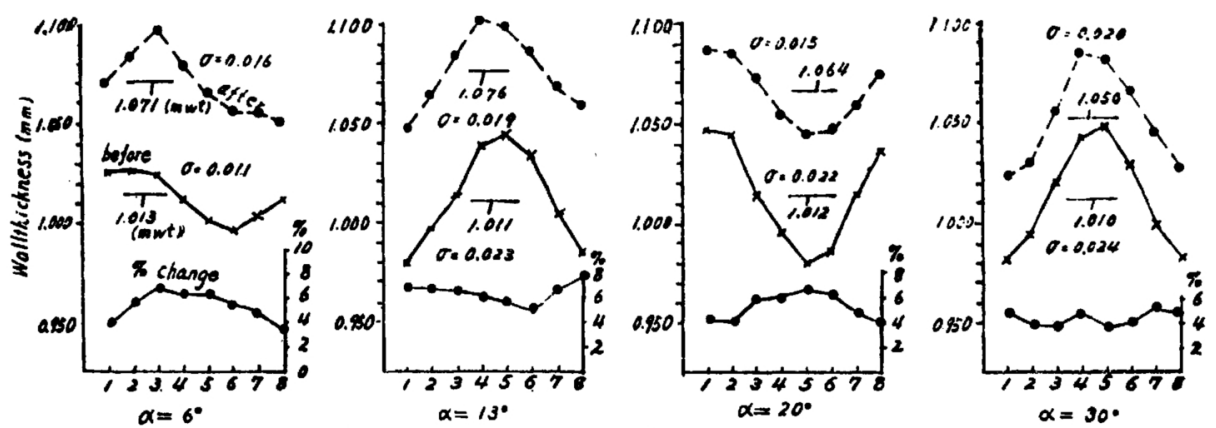

(c) $20.0 \phi \times 1.0 \longrightarrow 15.0 \mathrm{~mm} \phi 0 . \alpha$.
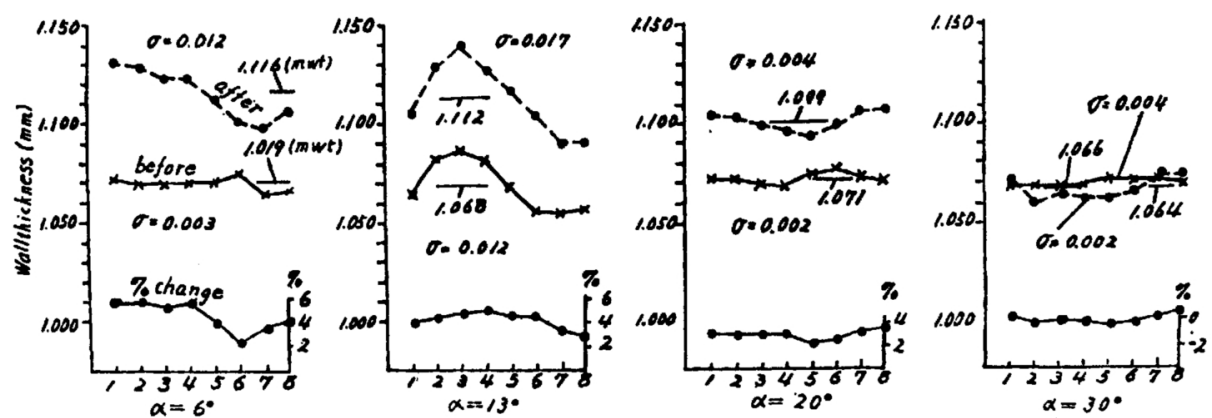

(d) $17.4 \phi \times 1.0 \longrightarrow 15.0 \mathrm{~mm} \phi 0 . d$.

Fig. 4 Wall-thickness changes by sinking hard copper tubes. 

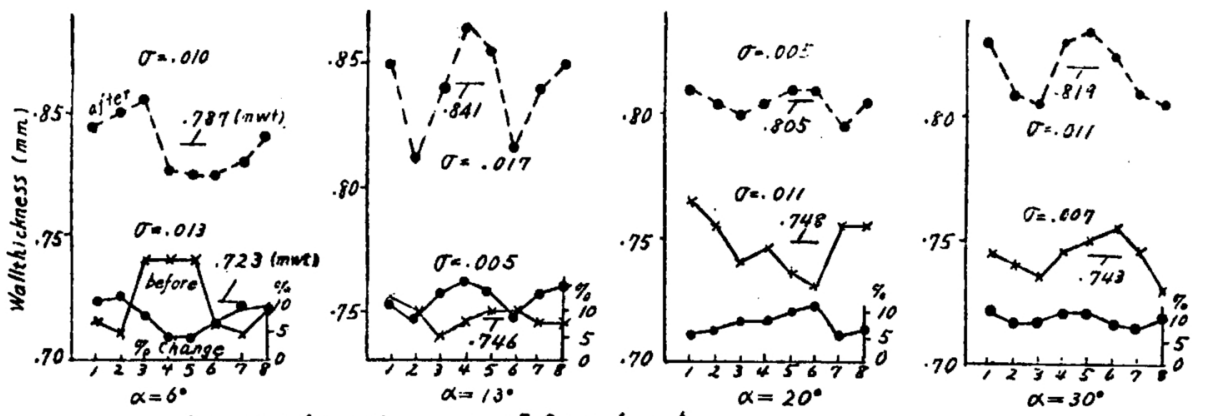

(a) $20.0 \phi \times 0.75 \longrightarrow 15.0 \mathrm{~mm} \phi 0 . \alpha$.

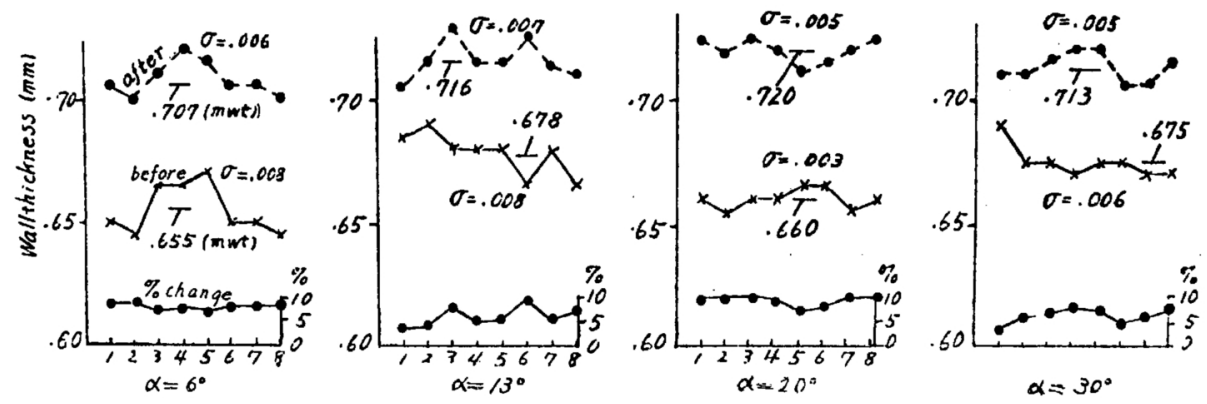

(b) $17.0 \phi \times 0.65 \longrightarrow 15.0 \mathrm{~mm} \phi 0 . \mathrm{d}$.

Fig. 5 Wall-thickness changes by sinking annealed copper tubes.
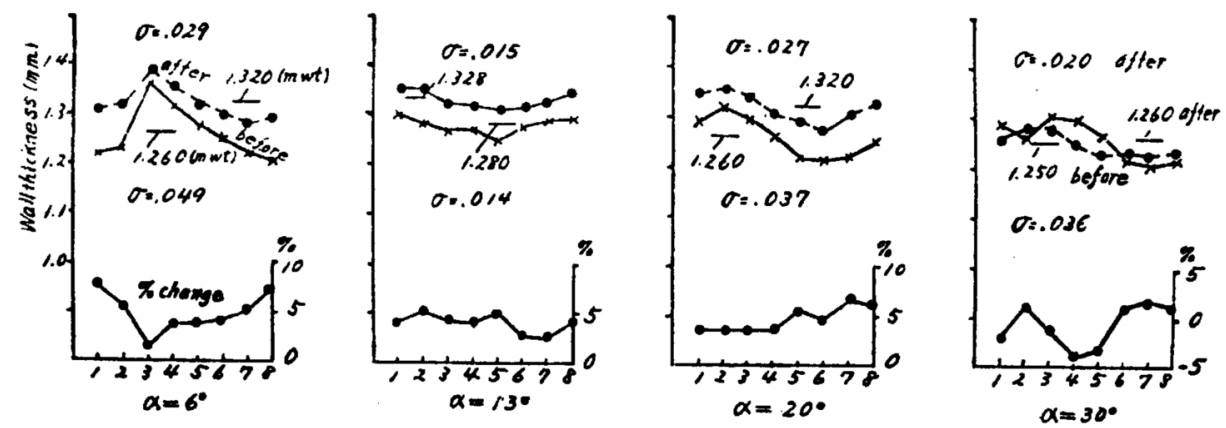

(a) $19.0 \phi \times 1.3 \ldots 15.0 \mathrm{~mm} \phi$ a.d. $(6951)$ annealed.
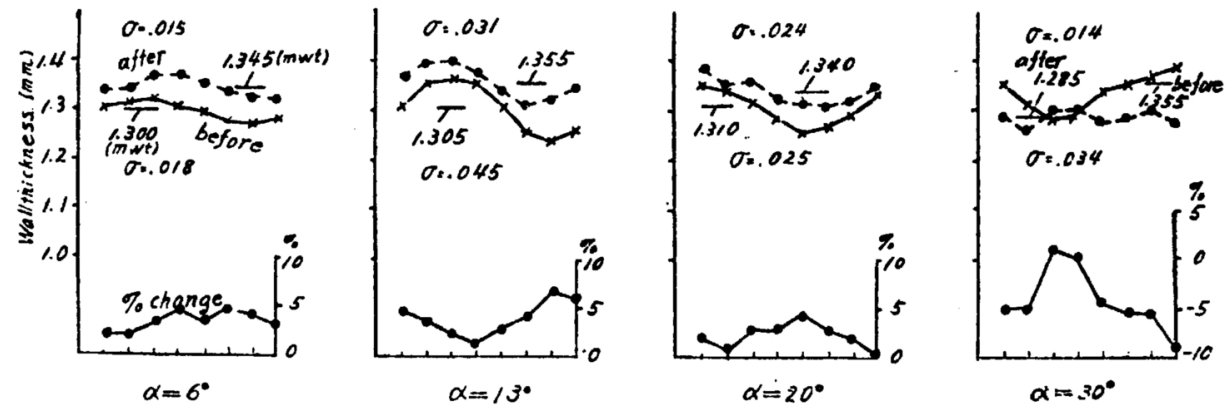

(b) $17.3 \phi \times 1.3 \longrightarrow 15.0 \mathrm{~mm} \phi$ o.d. (695i) anneaied.

Fig. 6 Wall-thickness changes by sinking $\mathrm{Al}$ alloy tube (6951). 
tribution curve, that is, decreasing the value in $\sigma$.

It was found after repeated experiments with more than fifty tubes of varying eccentricity (copper and aluminium alloys), that $\sigma$ could be decreased only when initial $\sigma$ was above $0.02 \mathrm{~mm}$. When initial $\sigma$ was below this value, that is, when the scatter was substantially small, $\sigma$ could not always be decreased but more often increased. This might be owing to the inevitable uneven sinking that took place along the tube-circumference. This has been proved true also with plug drawings, the results of which will be reported in a forthcoming paper.

In Fig. 7, all the values of $\sigma$ (before and after sinking) are shown in a simple form. The reading of $\sigma$ on the logarithmic abscissa is referred to the pre-drawn tube and that of on the ordinate to the sunk one. When $\sigma$ was not changed after sinking all the points plotted in the figure apparently lie on the $90^{\circ}$ - bisecting line and if $\sigma$ was changed, the points lie above or below the bisector according to whether $\sigma$ was increased or decreased.

It may be found, as mentioned above, that $\sigma$ could possibly be lowered by sinking only when it is somewhere above $0.02 \mathrm{~mm}$. Such a boundary value for $\sigma$ could be lowered still further by improving the drawing apparatus

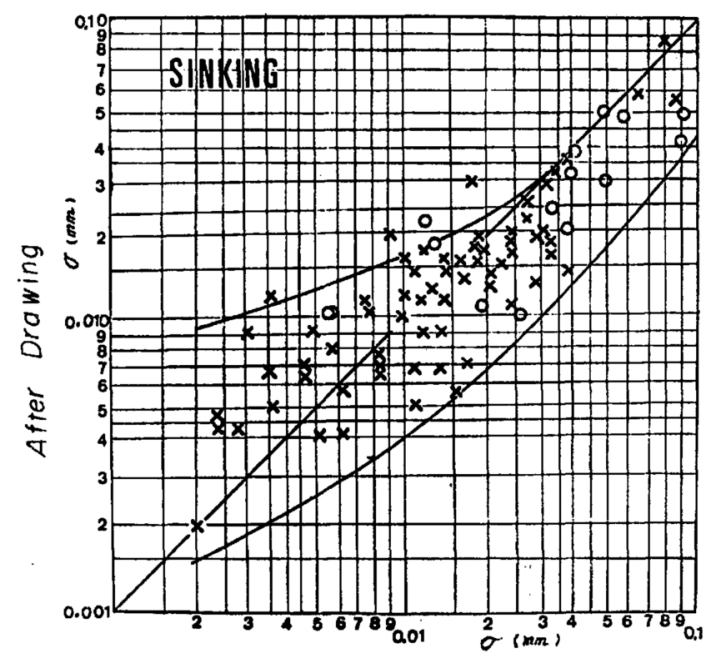

Before Drowing

Fig. 7 Standard deviations before and after hollowsinkings. $\times$ Copper tube, $\bigcirc \mathrm{Al}$ alloy tubes. so as to facilitate the tube-axis always being aligned with the die-axis during the sinking operation.

The points in Fig. 7 are from all the combinations of $\alpha$ and reductions as the scatter itself did not seem to be influenced by any valuable, such as reduction, die-taper, initial thickness, etc. The cross signs in the figure indicate the results from copper and circles from aluminium alloy tubes.

\section{Relationship between hardness distribu- tions before and after sinking}

The copper tubes of $17.0 \phi \times 0.65 \mathrm{~mm}$ were sunk to $15.0 \mathrm{~mm} \phi$ o.d. The hardness distributions on the transverse sections before and after drawing are shown in Fig. 8. The following points are worth noting:

(a) Every point in the pre-drawn wall increases in hardness by sinking, and the lower the initial hardness, the more is the increasing rate.

(b) Some of the points in the pre-drawn wall, particularly high in hardness, remain unchanged or even indicate some softening after they are drawn.

The softening in (b) is not a peculiar phenomenon in the tube drawing; it is in other cold workings ${ }^{(7)(8)}$. The phenomenon seems to explain the structural recovery either from the heat revolution by cold working or structural change due to the migration of dislocations. In either case, the hard portions seem more liable to be softened, although thorough explanations are still needed.

The same tendency was also observed by the aluminium alloy tube (6951).

\section{Dependence of consecutive sinkings on the} changes in wall thickness and eccentricity

Figure 9 shows step-to-step changes in mean wall thickness (mwt) and eccentricity ${ }^{\dagger}$ (ECC) when hard copper tubes, $20 \mathrm{~mm} \phi \times 1 \mathrm{~mm}$, were consecutively sunk by the four kinds of passing schedule (see Table 1).

For convenience' sake, $\sigma$ are also noted in

$\dagger$ Here ECC is the ratio of the great difference in wall thickness to the mean wall thickness in percent. 

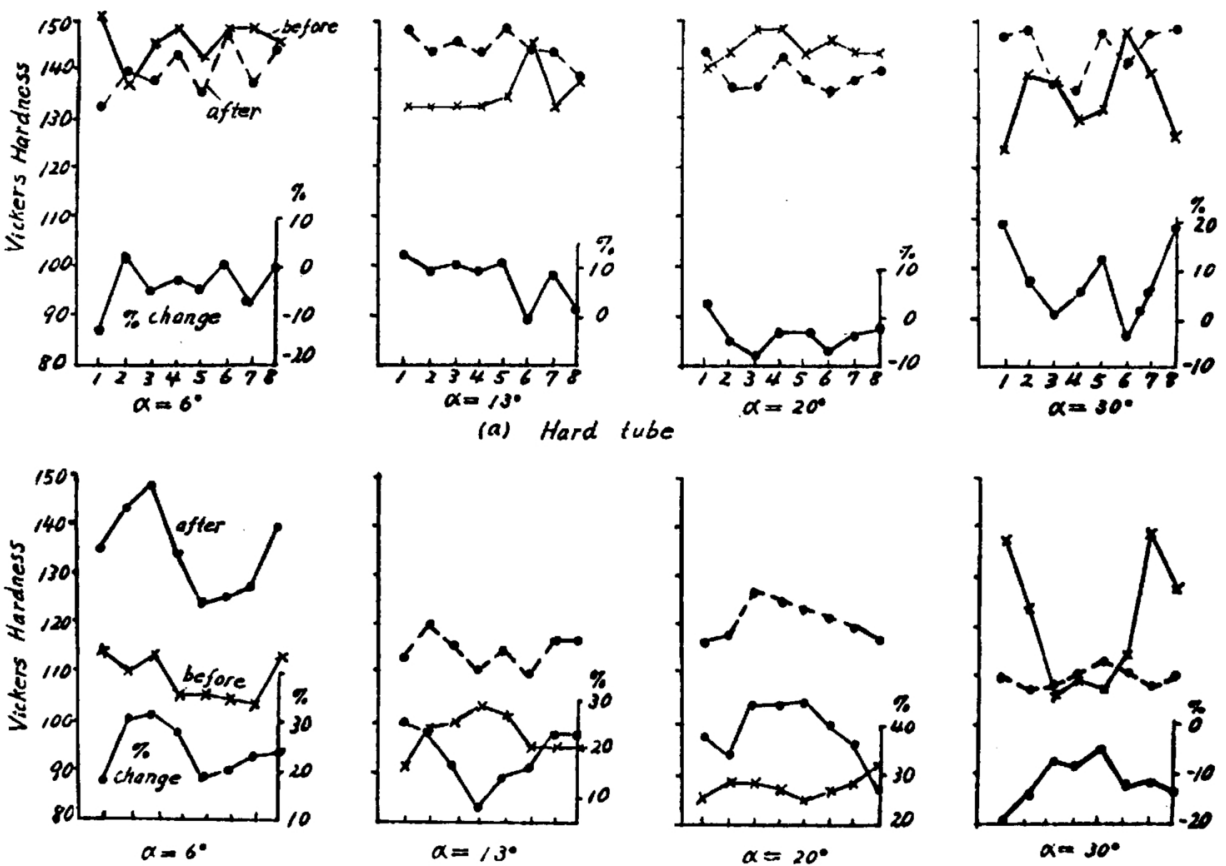

(b) Annealed tube

Fig. 8 Hardness changes by sinking copper tubes. $17.0 \phi \times 0.65 \rightarrow 15.0 \mathrm{~mm} \phi$ o.d.

Table 1 The four passing schedules.

\begin{tabular}{lllllll}
\hline \hline & \multicolumn{1}{c}{ Die-Pass } & \multicolumn{3}{c}{ Die-taper (degree) } \\
\hline 1. & $20 \rightarrow 13.0 \mathrm{~mm} \phi$ & - & 13 & 20 & - \\
2. $20 \rightarrow 17.4 \rightarrow 13.0 \mathrm{~mm} \phi$ & 6 & 13 & 20 & 30 \\
3. $20 \rightarrow 15.0 \rightarrow 13.0 \mathrm{~mm} \phi$ & 6 & 13 & 20 & 30 \\
4. $20 \rightarrow 17.4 \rightarrow 15.0 \rightarrow 13.0 \mathrm{~mm} \phi$ & 6 & 13 & 20 & 30 \\
\hline \hline
\end{tabular}

the figures. From the same data the changes in mwt are also correlated with die-taper and reduction and are shown in Fig. 10.

From each set of figures given, the followings may be pointed out.

(a) Unless reduction is not extremely high, the higher reduction tends more to increase the wall thickness (Fig. 10).

(b) The more the numbers of sinking pass under a fixed total reduction, the more the thickness increases (Fig. 9).

(c) High $\alpha$ tends to decrease the thickness, especially, when one-pass reduction is small (Fig. 10).

(d) The eccentricity can reasonably be lowered by consecutive sinking operations (Fig. 9), provided every die axis is always aligned with that of the tube, in short, when a tube is not misled through the die. Even a slight misleeding seemed to have a considerable effect on the wall-thickness deviation ${ }^{\dagger}$. This can be observed in Fig. 9 as abrupt increases in eccentricity in the course of consecutive sinkings.

It should be added here, that in the present experiments, although guide rings were placed before the dies, it was not easy to draw the tubes with their axes strictly being aligned with the die-axes. The tubes were sometimes badly misled into the die-hole, especially when $\alpha$ was large, thus causing abrupt increase in eccentricity which could not be decreased by the succeeded sinking operations.

\section{Conclusion}

From the experiments concerned the following conclusion is obtained:

(a) By hollow sinking, the initial wall thickness is varied, increased or decreased, by several percent. With small die-taper the

\footnotetext{
$\dagger$ This will be shown in the forthcoming paper.
} 

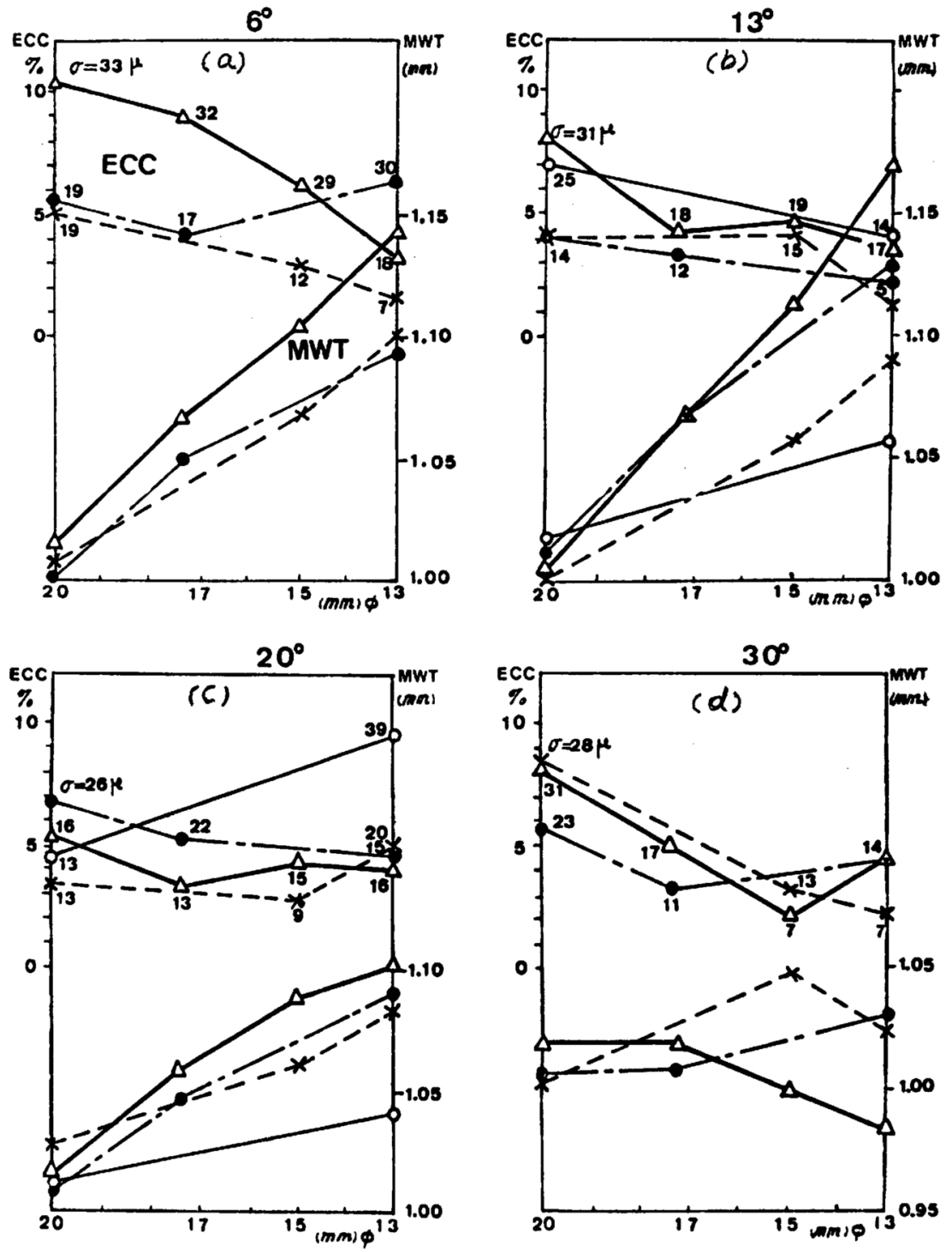

Fig. 9 Changes in mean wall thickness and eccentricity by consecutive sinking passes. Hard $\mathrm{Cu}$ tube.

thickness is usually increased, while with large taper (more than $20^{\circ}$ ) it is often decreased.

(b) If a tube with circumferential scatter in wall thickness (with eccentricity) is hollow sunk, the increasing rate in thickness is larger at the thinner portion of the wall. Accordingly, sinking seems to have a tendency to flatten the circumferential thickness-distribution curve, that is, to diminish the eccentricity of a tube. It was observed that if the eccentricity in terms of the standard deviation $\sigma$ of the pre-drawn tube is below $0.02 \mathrm{~mm}$, it is not decreased after sinking but increased sometimes. When $\sigma$ of the pre-drawn tube is above $0.02 \mathrm{~mm}$, it seems to be somewhat decreased.

(c) By repeating the sinking pass, the eccentricity can gradually be improved provided the tube-axis is always kept aligned with the die-axis.

(d) When the tube is reduced by hollowsinking, the hardness in the initial wall is increased by cold working in such a manner as 


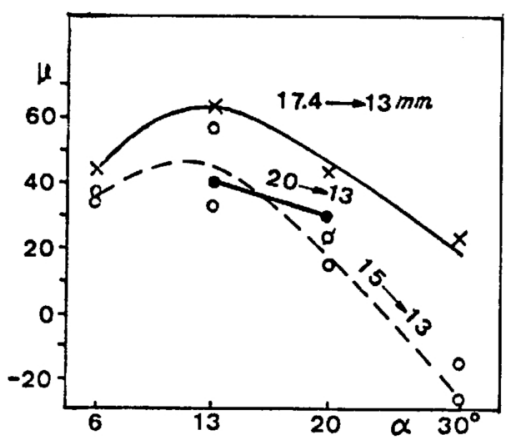

its rate is higher at the softer portion in the wall: the initial hard portions often exhibit some softening.

\section{Acknowledgments}

The author wishes to express his thanks to Furukawa Metals Manufacturing Co. and Furukawa Aluminium Co. for helpful discussions.

\section{REFERENCES}

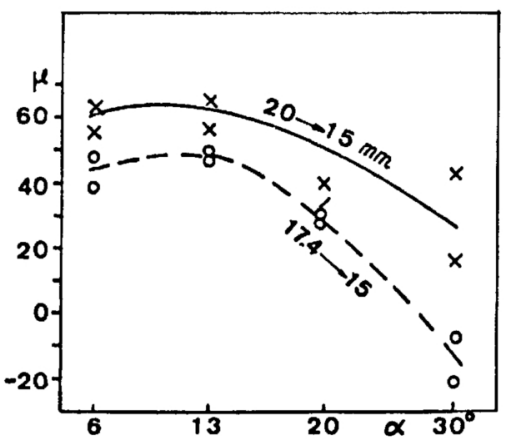

(1) S. Y. Chung et al.: J. Iron Steel Inst., 170 (1952), 29.

(2) T. Okamoto: Sumitomo Kinzoku, 6 (1954), 85.

(3) H. W. Swift: Phil. Mag., 11 (1949), 883.

(4) C. G. Moore and J. F. Wallace: J. Mech. Eng. Science, 3 (1961), 225

(5) J. E. Flinn: J. Basic Eng., ASM, (1970), 101.

(6) B. Avitzur: Metal Forming, McGraw-Hill, New York, (1968), p. 341.

(7) R. Grimes and J. C. Write: J. Inst. Metals, 96 (1968), 182.

(8) H. Tanaka and M. Sato: Trans. JIM, 1 (1960), 83.

Fig. 10 Thickness changes correlated with reduction and die-taper. 